\title{
Describing Words by Graphs
}

\author{
G. Congedo, G. Dimauro, S. Impedovo, G. Pirlo \\ Dipartimento di Informatica - Università di Bari \\ Via Amendola 173 - 70126 Bari - Italy
}

\begin{abstract}
In this paper a new technique for handwritten word description is presented. It is based on the development of a description graph which results little sensitive to handwriting variability. The experimental results show the effectiveness of the description graph in retaining the basic information for the recognition of the word image.
\end{abstract}

\section{Introduction}

Research on cursive word recognition has several fundamental applications in the development of automatic reading systems for bankchecks, tax forms, insurance forms and so on [1]. Unfortunately, it is commonly believed that cursive word recognition is a very complex task. This is due to the existence of many writing styles and to personal variability which depends on the physical and physiological condition of the writer as well as on the type of the supports used for writing (type of paper, pen or pencil etc.) [2]. .

Up to date, two different kinds of approaches have been considered for cursive word recognition [2,3]: analytical and wholistic. In both cases the recognition process strictly depends on the capability to obtain a suitable description of the word image: a very detailed description of the image can convey useless details which make the recognition process slower and less accurate; a poor description generally lacks of information useful for recognition.

In this paper a simple procedure for the description of handwritten words by graphs is proposed. The graphs, which retain fundamental morphological information of the word image, like oriented segments, concavities/convexities, loops and near-loops, are also used as input for a word recognizer based on an elastic pattern matching procedure. The experimental results demonstrate the effectiveness of the new technique in describing handwritten words for recognition aims.

\section{The Word Description Graph}

A cursive word is here considered as consisting of regular parts and singularities [2]. Regular parts are the segments of the word while singularities are the discontinuity regions. Three kinds of discontinuity regions are here considered: cross regions, bend regions and end regions. The cross regions are the intersection regions of segments; the bend regions are the adjacency regions of two segments with different orientation, the end regions are the extremity regions of a segment. 


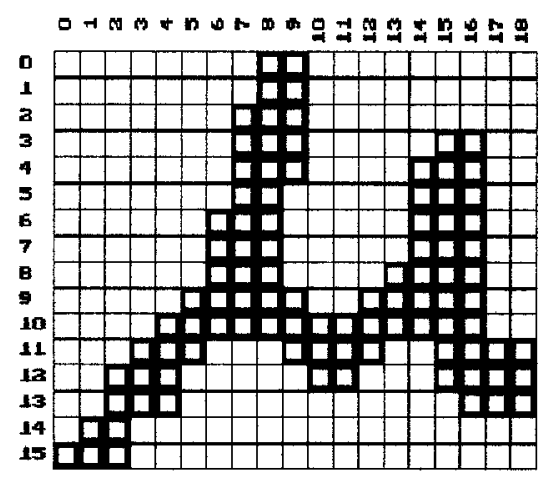

Figure 1

The description of a word can be accomplished by a description graph in which regular parts are represented by arcs while singularities are represented by nodes. The graph is realized by an automatic procedure which performs four different scanning on the word image according to four basic directions: horizontal, vertical, $-45^{\circ}$ and $+45^{\circ}$. The scanning in a specific direction produces a data structure containing the information about groups of adjacent pixels in the perpendicular direction. Specifically, the data structure is a list of sequences of nodes in which each node contains the information of the group of adjacent pixels found during the scanning process and the adjacency between groups on different rows is represented by pointers between the nodes. In each node the

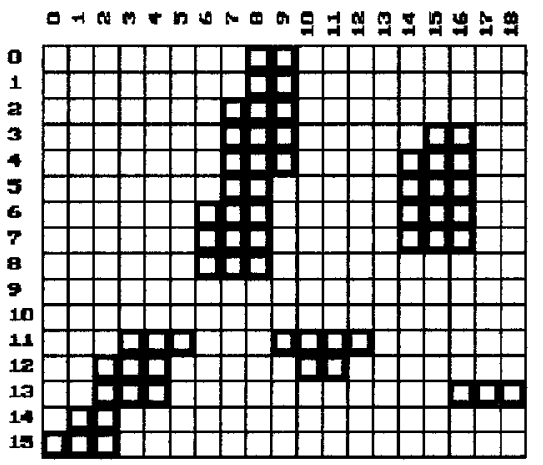

Figure 3

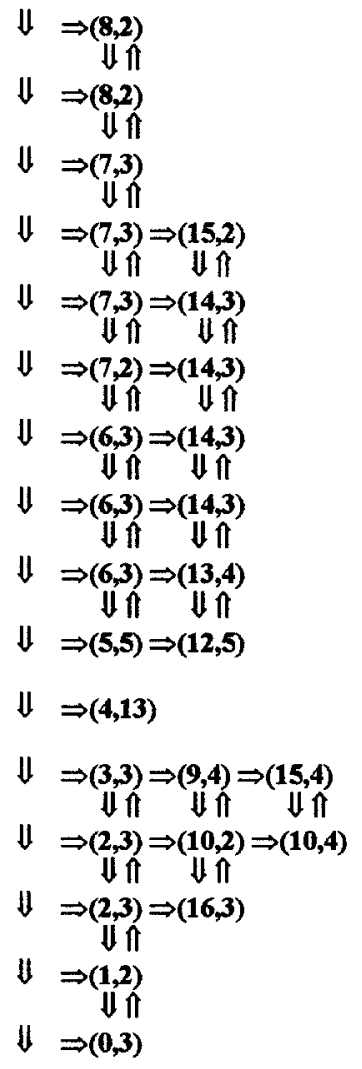

Figure 2 following information is stored: the first column of the group, the width of the group, the right pointer to the next group in the same row (if it exists ), the upper pointer to the adjacent group in the upper row (if it exists), the lower pointer to the adjacent group in the lower row ( if it exists). The upper and lower pointers are assigned according to simple rules: (1) two different nodes in the same row cannot point to the same node in the lower row; (2) the lower pointer of a node of a 
group which is adjacent to two or more groups in the lower row must be null. Figure 1 shows a simple example of a pattern. Figure 2 shows the corresponding data structure obtained by the horizontal scanning of the image. Each couple contains the column and the width of the corresponding group. The average width $w$ of the groups is then computed and each group whose width is greater than $w$ is removed as shown in figure 3 . Successively

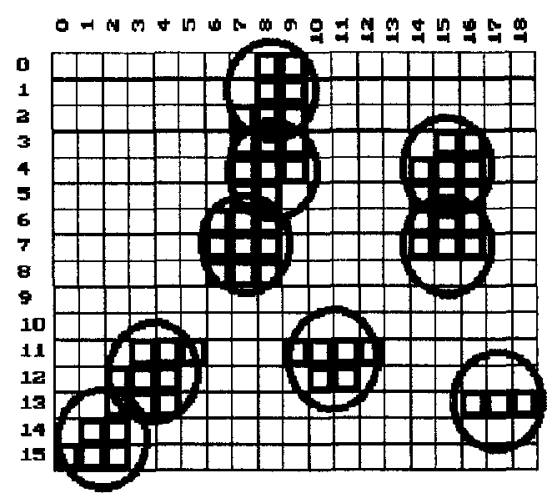

Figure 4 the writing units are identified. A writing unit is here defined as each set of $\mathbf{n}$ groups linked together in the vertical direction (by upper and lower pointers) for which $|\underline{w}-\mathbf{n}| \leq 1$, where $\mathbf{w}$ is the average width of the groups. Figure 4 shows that nine writing units are detected in the pattern in figure 1 . A sequence of adjacent writing units constitutes a segment. Blocks of one writing unit are removed and the direction of the remaining segments is computed in order to extract segments with vertical direction. Of course, the same process is repeated with other scanning directions in order to detect other segments. Figure 5A shows an example of handwritten word, figures 5B,C,D, and $E$ show respectively the detected segments with vertical, horizontal, $+45^{\circ}$ and $-45^{\circ}$ orientation.

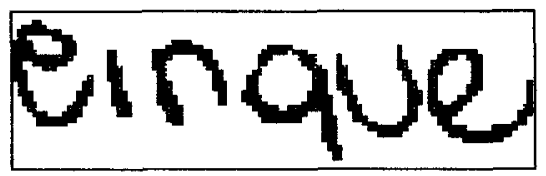

(A)

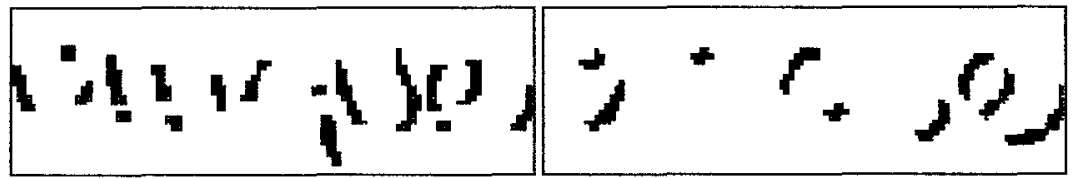

(B)

(D)

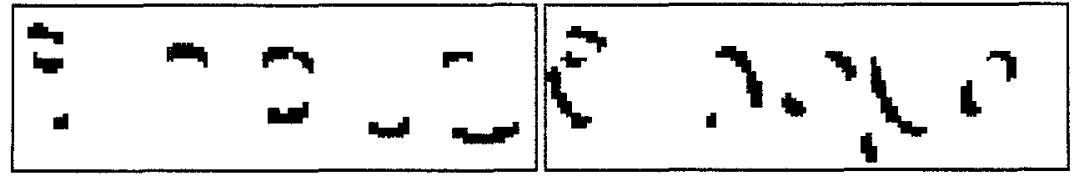

(C)

(E)

Figure 5 


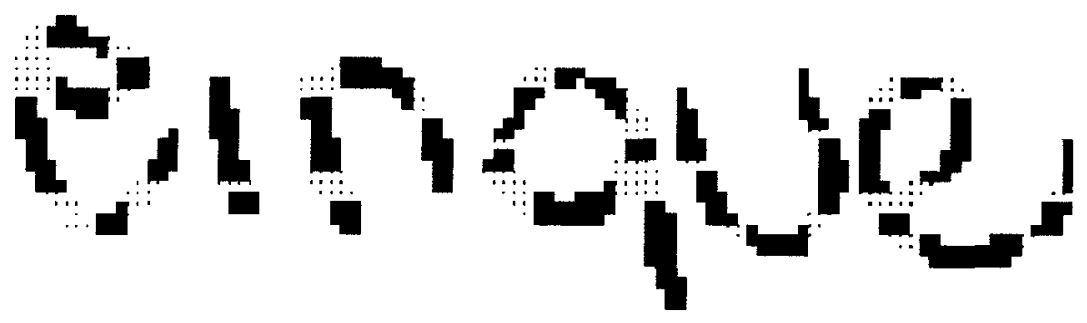

Figure 6

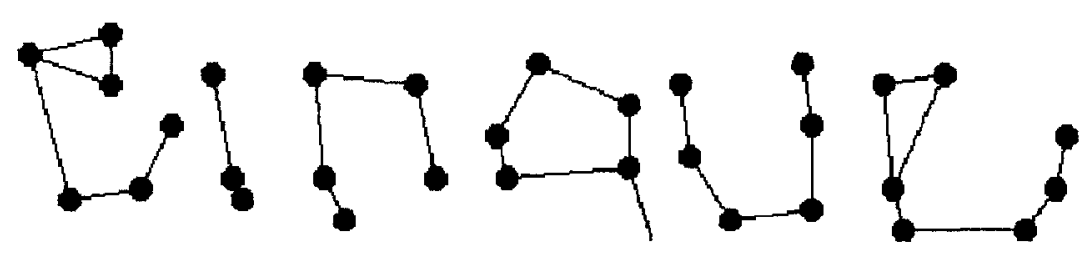

Figure 7

After that all the oriented segments have been detected, they are superimposed as figure 6 shows, and the adjacencies between segments are detected, obtaining the graph in figure 7 .

\section{Experimental Results}

In the first experimental phase, the description technique proposed in this paper has been compared with a traditional approach based on thinning. Figures 8 and 9 show two typical examples of handwritten words, the results of the Safe Point Thinning Technique (SPTA) [4], the results obtaind by and the proposed technique. It is easy to verify that the SPTA can yeld to unsatisfactory outcomes. This is the case of the spurious stroke shown in figure 8 and of the undesired loop shown in figure 9. Conversely, the new description technique leads to a graph which conveys fundamental information on the word shape without retaining useless details.

In the second experimental phase, the graphs have been used as input for an off-line word recognition system. Word recognition is based on finding specific structures in the graph like ascenders/descenders, loops, concavities/convexities, and simple oriented segments. In the graph of figure 7 the following sequence of structures has been detected: 1) convexity, 2) loop, 3) vertical segment, 4) concavity, 5) loop, 6) descender, 7) convexity, 8) loop, 9) convexity. Furthermore, for each structure the horizontal position of the barycentre is computed and the ordered sequence of structures is used for recognition. Word matching is carried out by an 
elastic matching procedure according to a suitable distance measure defined by an apriori analysis of the confusing structures.
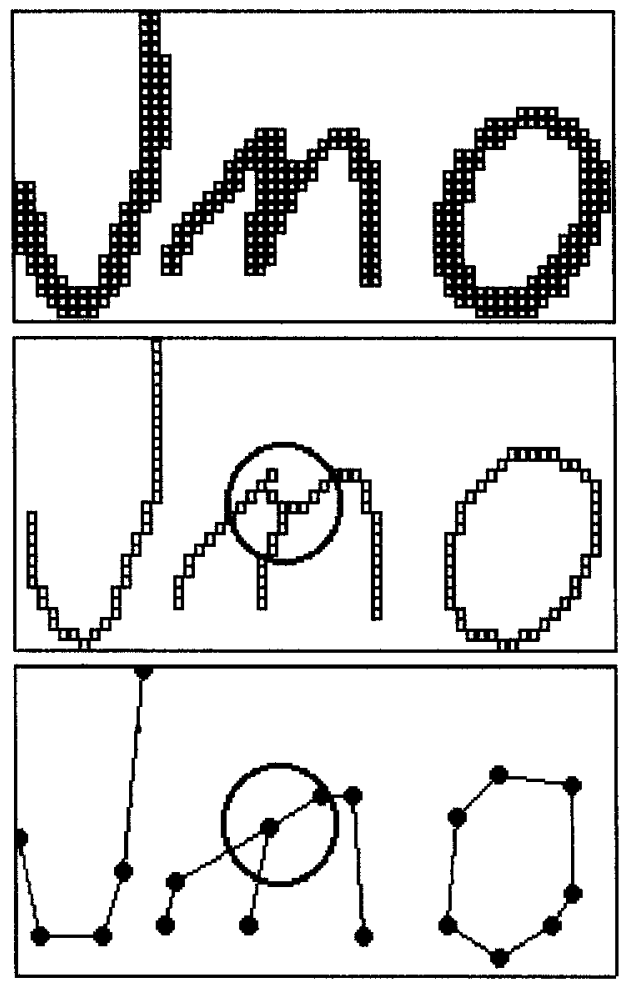

Figure 8

The experimental tests have been carried out in the domain of the 50 basic words of the worded amounts of italian bankchecks [5]. For this purpose, a reference database of 2000 words written by 40 writers and a test database of 2000 words written by further 40 writers have been used. For each test word a ranked list of candidates has been produced by the recognition system. On the entire test database the following results have been obtained: $69 \%$ of correct recognition in the first position of the list; $78 \%$ of correct recognition in the first two positions of the list; $79 \%$ of correct recognition in the first three positions of the list.

\section{Conclusion}

In this paper a useful technique is proposed for the description of handwritten word by graph. The experimental results show that the new technique is able to retain morphological information on the word shape without retaining usefulness details. 

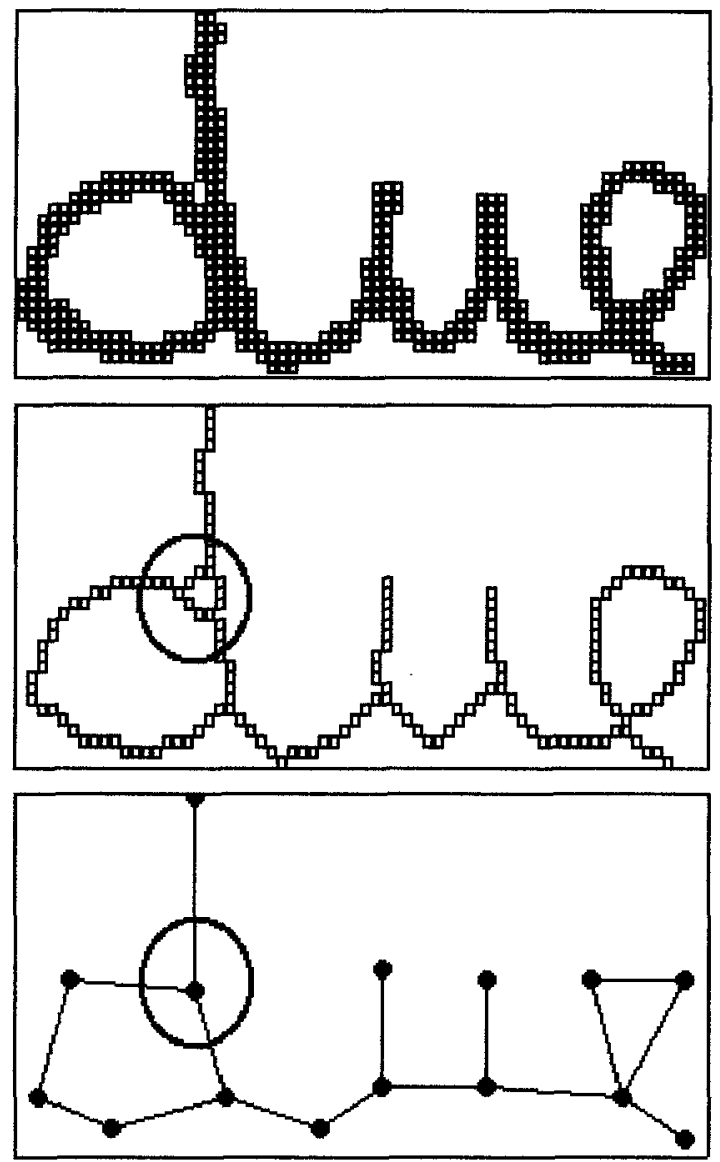

Figure 9

\section{References}

1. K. Sakai, H. Asami, Y. Tanabe, "Advanced application systems for handwritten character recognition", in From Pixels to Features III - Frontiers in Handwriting Recognition, S. Impedovo, J.C. Simon, (eds.), Elsevier Publ., 1992, pp. 175-186.

2. E. Lecolinet, O. Baret, "Cursive Word Recognition: Methods and Strategies", in Fundamentals in Handwriting Recognition, S. Impedovo (ed.), NATO ASI Series, Springer Verlag 1994, pp. 235-263.

3. J.Hull, T.-K. Ho, J. Favata, V. Govindaraju, S. Srihari, "Combination of Segmentationbased and Wholistic Handwritten Word Recognition Algorithms", in From Pixels to Features III - Frontiers in Handwriting Recognition, S. Impedovo, J.C. Simon, (eds.), Elsevier Publ., 1992, pp.229-240.

4. N.J. Naccache, R. Shingal, "SPTA:A proposed algorithm for thinning binary Pattems", IEEE Trans. Syst. Man, Cybern., no. 3, 1984.

5. G. Dimauro, M.R. Grattagliano, S. Impedovo, G. Pirlo, "A System for Banckchecks Processing", IEEE Computer Society Press, Vol. 4960-02, ICDAR '93, Tsukuba Science City -Japan, Oct. 1993, pp. 454-459. 